\title{
La petite hydroélectricité dans la politique française des énergies renouvelables
}

\author{
Small hydropower in the context of French renewable energy policy
}

\author{
par F. Armand \\ Agence de l'Environnement et de la Maîtrise de l'Energie (ADEME)
}

Renewable Energy sources are available for a large development in the world. They are local sources, their technologies are mature and make no gas pollution. There is an important potential and only a little part of it is exploited. Referring to small hydro, 500 TWh are available and only 80 are harnessed.

In France, there are 2500 professionals, and the turnover of consultants and industrials (excluding the sale of electricity) is about 400 M FFR.

Within an international context where countries try to reduce pollution and the emission of greenhouse effect gases, the French government decided to relaunch energy savings and renewable energy sources. Regarding small hydro, it is planned to develop environment friendly operations : refurbishment, upgrading, environmental labels and also to lend a support to export.

\section{L L'AVENIR DES ÉNERGIES RENOUVELABLES}

Les énergies renouvelables sont les premières sources d'énergie utilisées par l'homme. A l'heure actuelle, l'énergie hydraulique n'intervient plus que pour $6 \%$ et le bois pour $7,5 \%$ dans le bilan énergétique mondial, le reste étant produit par les énergies fossiles (charbon, pétrole, gaz, uranium).

Mais, diverses menaces pèsent sur l'avenir des énergies fossiles : diminution des réserves et augmentation des coûts d'exploitation de minerais moins accessibles, pollution résultant de la combustion, contribution des gaz à l'effet de serre, risques inhérents à la điffusion des technologies nucléaires et problèmes soulevés par le traitement des déchets, etc.

Les énergies renouvelables quant à elles, offrent de vastes possibilités de développement. Ces énergies sont locales ; elles utilisent des technologies maintenant bien rodées et n'entraînent pas de pollution gazeuse. D'autre part, le gisement n'est encore que très peu exploité. En petite hydroélectricité (PHE), on estime le potentiel de sites rentables à $500 \mathrm{TWh}$ par an dont seulement $80 \mathrm{TWh}$ sont actuellement exploités.

Dans la gamme des différentes solutions qui peuvent être apportées par les énergies renouvelables, la PHE répond à des demandes bien spécifiques, en fonction notamment des gisements disponibles et des puissances à installer.

\section{II — LA PETITE HYDROÉLECTRICITÉ EN FRANCE}

\subsection{Quelques chiffres}

$\mathrm{Si}$ on adopte la classification de l'UNIPEDE (Union Internationale des Producteurs et Distributeurs d'Energie Electrique), sont considérées comme petites centrales les installations de puissance installée inférieure à $10 \mathrm{MW}$. Leur production annuelle est évaluée en France à 7,5 TWh dont une moitié environ est fournie par EDF, la SNCF, la CNR, les régies municipales, l'autre moitié étant assurée par environ 1500 producteurs indépendants.

La production électrique de ces petites centrales génère un chiffre d'affaires de plus de 2 milliards de francs. Quant aux travaux de réalisation, réhabilitation, maintenance des installations, leur chiffre d'affaires est d'environ $400 \mathrm{MF}$ à $90 \%$ à l'export.

La profession compte environ 2500 emplois.

\section{- 2.2 Les contraintes}

On sait que, malgré les avantages tant écologiques que technico-économiques présentés par cette forme d'énergie, la petite hydroélectricité ne connaît pratiquement plus aucun développement dans notre pays.

Cette situation est essentiellement due aux raisons suivantes :

- les meilleurs sites ont évidemment été équipés les premiers ; 
- les fédérations de pêche et certaines associations de défense de l'environnement s'opposent vigoureusement à la mise en place de nouveaux équipements hydroélectriques ;

- divers décrets de classement pris dans un souci de préservation de l'écologie de la rivière interdisent l'usage énergétique de l'eau sur un nombre important de rivières et de tronçons de rivière du pays.

Compte tenu d'une part du niveau des tarifs d'achat de l'électricité et d'autre part des dépenses supplémentaires et des baisses de revenus entraînées par le respect de la réglementation relative aux impacts sur l'environnement, l'équilibre économique des projets devenait difficile à assurer.

\section{III $\square$ LES ACTIONS ENTREPRISES}

\subsection{La tarification}

En ce qui concerne les tarifs d'achat par EDF de l'électricité aux producteurs indépendants, une négociation entre les pouvoirs publics, EDF et les producteurs a conduit à un accroissement des tarifs pour les installations récentes et en cas d'augmentation de puissance des installations. Cette mesure devrait donc apporter une part de réponse aux difficultés énumérées ci-dessus.

\subsection{Les impacts sur l'environnement}

Dans un contexte budgétaire très difficile, l'ADEME qui est chargée des deux missions de soutien des énergies renouvelables et de préservation de l'environnement a souhaité prioritairement contribuer à l'analyse des impacts des petites centrales sur l'environnement et la recherche de solutions pour y remédier. Dans ce but, elle a participé avec ses partenaires de la filière à différentes études :

- rédaction du guide sur l'intégration des petites centrales dans l'environnement avec comme partenaires : Société Hydrotechnique de France, Ministère de l'Environnement, CNR, EDF, Conseil Supérieur de la Pêche (CSP), CEMAGREF ;

- suivi expérimental hydrologique et hydrobiologique de sites de petites centrales, pour différentes valeurs du débit réservé, partenaires : EAF, Hydro-M :

- évolution des peuplements piscicoles sur des sites de petites centrales, partenaires : CSP, CEMAGREF ;

- campagne de mesures sur le bruit des petites centrales et rédaction d'un guide de recommandations ; partenaires : CETIM, turbiniers :

- étude de l'impact sur la rivière des lubrifiants utilisés dans les petites centrales, partenaires : CETIM, turbiniers.

D'autre part, l'ADEME réfléchit avec ses partenaires à un projet de label environnement à décerner sur demande à des petites centrales répondant à certains critères environnementaux.

\subsection{Formation - information}

Un programme comportant des modules de formation et d'information, ainsi qu'un réseau d'assistance a été monté dans le cadre d'un programme ALTENER avec comme leader l'ENSHMG (Ecole Nationale Supérieure d'Hydraulique et de Mécanique de Grenoble) et la participation d'industriels, de formateurs et l'ADEME.

Le travail de mise en place est terminé et cette opération entre maintenant dans sa phase opérationnelle. Les demandes de formation, information, assistance vont donc désormais pouvoir être prises en charge dans ce cadre.

\section{- 3.4 Action internationale}

Au niveau international, I'ADEME participe au groupe de travail sur la petite hydroélectricité dans le cadre d'un accord de coopération sur les technologies hydroélectriques de l'Agence Internationale de l'Energie (AIE) pour l'énergie hydraulique. Les résultats attendus de ce travail sont :

- mise au point d'une banque de données sur Internet

- rassemblant les informations disponibles sur les petites centrales existantes et les gisements exploitables en petite hydroélectricité à travers le monde :

- accueillant des échanges d'informations sur les programmes et activités des pays.

- données de synthèse sur les méthodes d'estimation de sites, les technologies à coût réduit, la $R \& D$, les outils financiers.

Ces travaux sont en cours. La parution des documents est prévue pour 1999

\section{IV — PROPOSITION DE RELANCE}

\subsection{L'opportunité actuelle}

Une relance de la maîtrise de l'énergie a été décidée par le gouvernement. Dès l'année prochaine, elle doit se traduire concrètement par une augmentation substantielle des crédits pour les économies d'énergie et les énergies renouvelables.

L'ADEME a conduit une réflexion sur l'ensemble des secteurs concernés.

Pour la petite hydroélectricité, l'accent a surtout été mis sur l'amélioration des équipements existants. En effet, de nombreux seuils existants peuvent être équipés avec des impacts minimes sur la rivière, des installations en place peuvent être réhabilitées et leur productivité améliorée. De tels projets sont à mettre au point en concertation entre les principaux acteurs concernés : ministère de l'Environnement et ses services décentralisés, Agences de l'Eau, ADEME, Conseil Supérieur de la Pêche, bureaux d'études, producteurs autonomes, etc., et à mener en commun avec des actions concernant également d'autres usagers de la rivière : entretien des berges et du seuil, pisciculture, etc.

Les axes suivants ont été retenus pour les interventions de I'ADEME :

- aide à la décision pour des réhabilitations,

- optimisation de l'existant,

- labellisation environnementale.

D'autre part, l'activité des instriels du secteur étant tournée principalement vers l'export, il est prévu également une aide au montage de projets à l'international. 


\subsection{Voies de développement}

Voici quelques idées directrices pour la mise en æuvre de ces actions au niveau national.

Plutôt que de travailler sur des sites isolés, on viserait des opérations groupées sur des rivières ou tronçons de rivières.

Les aspects énergétiques sont à regarder en liaison avec les autres aspects de la rivière : régulation des débits et niveaux, entretien des berges et seuils, loisirs, pêche, tourisme, etc.

Les projets sont à conduire en étroite collaboration avec les autres acteurs, dans le cadre des SDAGE et peuvent s'inscrire dans des contrats de rivière ou dans des SAGE, ce qui implique un partenariat particulier avec les Agences de l'Eau.

Des sites déjà identifiés pourront rapidement être l'objet d'opérations-pilotes. En même temps pourra être entreprise une recherche d'autres sites.

\subsection{Contexte international}

Ce programme de travail s'inscrit dans les objectifs de développement des énergies renouvelables préconisés par les conférences internationales sur la sauvegarde de l'environnement ainsi que par le Livre Blanc de la Commission Européenne sur les sources d'énergie renouvelables. 